\title{
M. tuberculosis Gene Expression during Transition to the "Non-Culturable" State
}

\author{
E.G. Salina', H.J. Mollenkopf ${ }^{2 *}$, S.H.E. Kaufmann², A.S. Kaprelyants ${ }^{1}$ \\ 'A.N. Bach Institute of Biochemistry, RAS, 119071, Moscow, Leninsky pr., 33, fax (495) 954-27-32, \\ elenasalina@mail.ru \\ ${ }^{2}$ Max Planck Institute for Infection Biology, 10117, Berlin, Chariteplatz 1, fax 49-30-284 60501 , \\ *E-mail: mollenkopf@mpiib-berlin.mpg.de
}

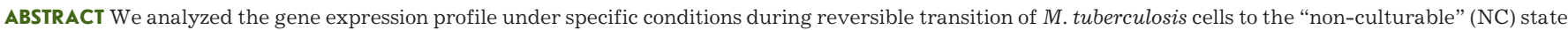

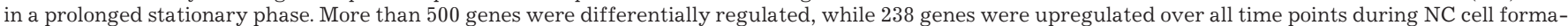

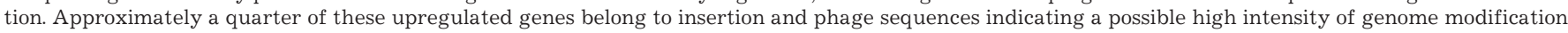

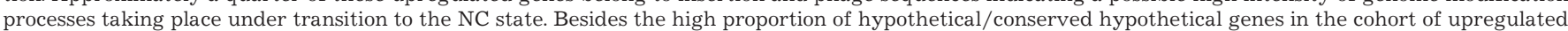

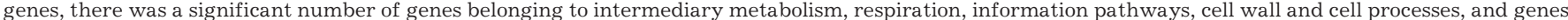

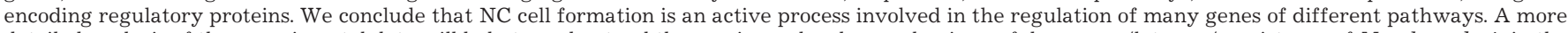

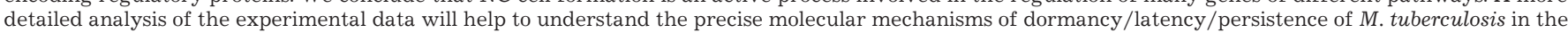

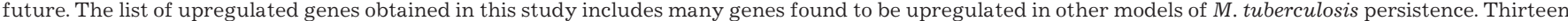

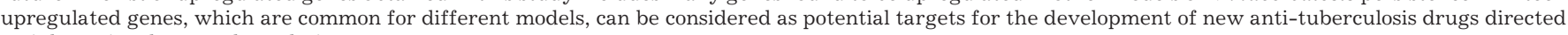
mainly against latent tuberculosis.
\end{abstract}

Keywords: $M$. tuberculosis, latent tuberculosis, "non-culturable" cells, global gene expression profile

\section{INTRODUCTION}

Mycobacterium tuberculosis - the causative agent of tuberculosis - can persist in the human host for decades after infection. Such a latent M. tuberculosis state is traditionally connected with its transition to the dormant state, accompanied by loss of culturability [1]. This makes it practically impossible to reveal latent infection by traditional biochemical and microbiological means and attempt to cure it by antibiotic therapy. To study latent infection in live organisms, several modifications of the experimental model of dormancy during hypoxia in vitro are used [2, 3]. However, none of them imitates such an important state of bacteria as their "non-culturability" in the dormant state. We have established an experimental model where dormant $M$. tuberculosis cells are "non-culturable" (NC) and can reactivate under special conditions [4].

To reveal the biochemical processes accompanying the transition of bacteria to the NC state and to understand the mechanisms of this phenomenon, we analyzed $M$. tuberculosis gene expression profile during the formation of NC cells.

\section{METHODS}

M. tuberculosis total RNA samples were extracted from cells in the late logarithmic phase (5 days of cultivation) and during the transition of cells to the NC state under incubation in the stationary phase at different time points $(21,30$, 41 and 62 days of cultivation) as described previously [5]. cDNA was generated from $1 \mu \mathrm{g}$ RNA using random hexamers and reverse transcriptase (Superscript III, Invitrogen, Karlsruhe, Germany) according to the manufacturer's instructions. Reverse transcribed samples were purified with the QIAquick PCR purification kit (Qiagen, Hilden,
Germany) and labeled with Cy3- and Cy5-ULS according the suppliers' recommendations (Kreatech Diagnostics, Amsterdam, The Netherlands). Finally, labeled samples were purified with KREApure spin columns. Microarray experiments were performed as dual-color hybridizations. In order to compensate for the specific effects of the dyes and to ensure statistically relevant data, a color-swap dye-reversal analysis was performed. Cy3-labeled cDNA (250ng) corresponding to cells from different time points in the stationary phase was competitively hybridized with the same amount of Cy5-labeled cDNA of the control sample as color-swap technical replicates onto self-printed microarrays comprising a collection of 4,325 M. tuberculosis-specific "Array-Ready" 70mer DNA oligonucleotide capture probes and 25 control sequences (Operon Biotechnologies, Koeln, Germany) at $42^{\circ} \mathrm{C}$ for $20 \mathrm{~h}$. Arrays were washed 3 times using a SSC wash protocol followed by scanning at $10 \mu \mathrm{m}(\mathrm{Mi}-$ croarray Scanner BA, Agilent, Technologies, Waldbronn, Germany). Image analysis was carried out with Agilent's feature extraction software version (Agilent, Technologies, Waldbronn, Germany). The extracted MAGE-ML files were further analyzed with the Rosetta Resolver Biosoftware, Build 7.1 (Rosetta Biosoftware, Seattle, USA). Ratio profiles comprising color-swap hybridizations were combined in an error-weighted fashion to create ratio experiments. Anticorrelation of dye-reversals was determined by the compare function of Resolver. Next we applied a Student's t-test. Finally, by combining a 1.5 -fold change cutoff to ratio experiments and the anticorrelation criterion together with the signatures from the Student's t-test, all valid data points had a $\mathrm{P}$-value $<0.01$, rendering the analysis highly robust and reproducible. 


\section{RESULTS AND DISCUSSION}

We found earlier that $M$. tuberculosis cultivation in the modified Sauton medium without $\mathrm{K}^{+}$supplemented by dextrose, BSA, and sodium chloride led to a decrease in colony forming units (CFU) on the solid medium in the stationary phase [4]. After 60 days of cultivation, the CFU count dropped to $10^{5}$ per $\mathrm{ml}$ (Fig. 1), which meant a transition of $99.9 \%$ of cells to the NC state. During further cultivation of cells, spontaneous recovery of NC cells was observed. It is important that the NC state was reversible, and that cells with a minimum CFU count could be reactivated after regrowing them in fresh medium.

Comparison of the gene expression profile at different time points from the stationary to the logarithmic phase (5-day cultivation) revealed a different expression (at least 1.5-fold) for a significant number of genes (566), which corresponds to $14 \%$ of the M. tuberculosis genome. Some 238 genes are upregulated and 237 downregulated over all time points during the entire culture period. Table 1 shows the functional category of differentially regulated genes during the transition of cells to the NC state.

Besides the significant amount of conserved hypotheticals/unknown genes, many genes involved in the intermediary metabolism and respiration, virulence, detoxification and adaptation, lipid metabolism, information pathways, cell wall and cell processes were downregulated.

A considerable amount of genes coding hypothetical proteins were also found to be upregulated in the NC state: remarkably, genes encoding insertion sequences and phages represented about a quarter of the genes upregulated in the NC state, whereas their proportion in the genome was smaller - only $3.7 \%$. This fact is a possible illustration of the high intensity of genome modification processes during the transition of cells to the NC state.

A significant proportion of upregulated genes belonged to the intermediary metabolism and respiration category, in par-

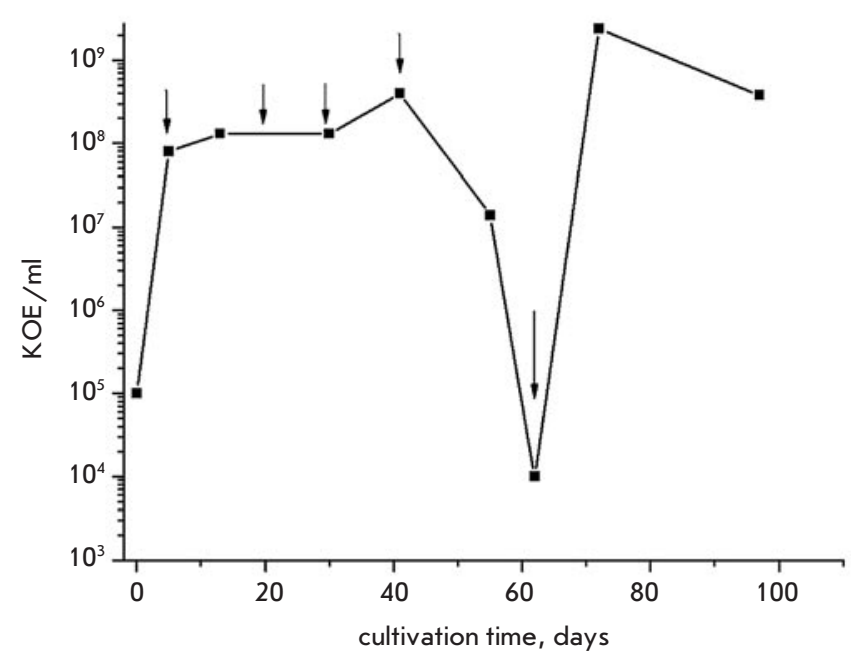

Fig. 1. Formation of NC M. fuberculosis cells in the stationary phase. Time points where RNA was isolated are marked by arrows

ticular, gcvB and ald, coding, respectively, glycine dehydrogenase and L-alanine dehydrogenase, proteases pepR and clpC2. $i c l 1$ - one of the genes coding isocitrate lyase, anaplerotic enzyme, existing in the M. tuberculosis cells in two isoforms icl 1 and icl 2 - was found upregulated. Isocitrate lyase is the key enzyme of the glyoxylate cycle - a metabolic pathway, which is an alternative for the tricarboxylic acid cycle and allows the synthesis of carbohydrates from simple precursors. In particular, it plays an important role in seed germination, where fatty acids are used as the main storage of carbon and energy. The induction of some genes involved in lipid degradation, such as fadD9, fadE24, fadE26, and fatty acid degradation, sco $A$, is indicative of the active role of the glyoxylate cycle in NC cells already found for the Wayne persistence model [2].

Table 1. Functional categories of $M$. tuberculosis genes with changed expression level during transition to the NC state

\begin{tabular}{|c|c|c|c|c|c|}
\hline \multirow{2}{*}{ Functional categories } & \multicolumn{2}{|c|}{$\begin{array}{l}\text { Genes induced during } \\
\text { transition to the NC state }\end{array}$} & \multicolumn{2}{|c|}{$\begin{array}{l}\text { Genes repressed during } \\
\text { transition to the NC state }\end{array}$} & \multirow{2}{*}{$\begin{array}{l}\text { Percent } \\
(\%) \text { in the } \\
\text { genome }\end{array}$} \\
\hline & $\begin{array}{l}\text { Number of } \\
\text { genes }\end{array}$ & $\%$ & $\begin{array}{l}\text { Number of } \\
\text { genes }\end{array}$ & $\%$ & \\
\hline Virulence, detoxification, adaptation & 5 & 2.1 & 7 & 2.9 & 2.6 \\
\hline Lipid metabolism & 6 & 2.5 & 20 & 8.4 & 5.9 \\
\hline Information pathways & 13 & 5.5 & 23 & 9.7 & 5.8 \\
\hline Cell wall and cell processes & 24 & 10.1 & 59 & 24.8 & 18.8 \\
\hline Insertion sequences and phages & 58 & 24.4 & 1 & 0.4 & 3.7 \\
\hline $\mathrm{PE} / \mathrm{PPE}$ & 7 & 2.9 & 11 & 4.6 & 4.2 \\
\hline Intermediary metabolism and respiration & 42 & 17.7 & 50 & 21.1 & 22.4 \\
\hline Regulatory proteins & 16 & 6.7 & 4 & 1.7 & 4.8 \\
\hline Unknown/hypothetical & 67 & 28.1 & 63 & 26.5 & 31.9 \\
\hline Total number of genes & 238 & - & 237 & - & $3924 / 100$ \\
\hline
\end{tabular}


Table 2. Significantly upregulated genes during transition to the NC state in the stationary phase

\begin{tabular}{|c|c|c|c|c|c|c|c|}
\hline \multirow[b]{2}{*}{ ORF } & \multirow[b]{2}{*}{ Gene } & \multirow[b]{2}{*}{ Gene product } & \multicolumn{5}{|c|}{ Change of gene expression level } \\
\hline & & & 5 days & 21 days & 30 days & 41 days & 62 days \\
\hline Rv0186 & $b g l S$ & Beta-glucosidase & 1 & 4.20459 & 8.33686 & 6.51867 & 5.24295 \\
\hline Rv0841c & & Transmembrane protein & 1 & 31.11093 & 52.56174 & 13.79488 & 11.85425 \\
\hline Rv0989c & $\operatorname{grcC} 2$ & Polyprenil-diphosphate synthase & 1 & 7.60797 & 6.29748 & 7.58723 & 3.94285 \\
\hline Rv1369c & & Transposase & 1 & 3.17178 & 3.9213 & 4.22925 & 3.86883 \\
\hline Rv1394c & cyp132 & Cytochrome P450 132 & 1 & 8.89047 & 7.50161 & 3.72981 & 3.12534 \\
\hline Rv1395 & & Transcriptional regulatory protein & 1 & 3.22394 & 11.65875 & 7.03908 & 4.27327 \\
\hline Rv1397c & & Conserved hypothetical protein & 1 & 6.95276 & 11.79184 & 5.97336 & 5.77752 \\
\hline Rv1460 & & Transcriptional regulatory protein & 1 & 3.87617 & 5.50637 & 6.90405 & 3.78332 \\
\hline Rv1831 & & Hypothetical protein & 1 & 3.1468 & 5.74692 & 5.14019 & 4.04747 \\
\hline Rv1991c & & Conserved hypothetical protein & 1 & 4.04696 & 4.12618 & 4.06786 & 4.65579 \\
\hline Rv1992c & $\operatorname{ctpG}$ & Metal cation transporter ATPase & 1 & 5.2883 & 7.31348 & 4.7442 & 4.22806 \\
\hline Rv2106 & & Transposase & 1 & 3.01418 & 5.61324 & 4.77882 & 5.09925 \\
\hline Rv2254c & & Integral membrane protein & 1 & 7.09534 & 6.53956 & 3.33899 & 4.63885 \\
\hline Rv2278 & & Transposase & 1 & 3.28663 & 6.78129 & 6.28036 & 4.13102 \\
\hline Rv2354 & & Transposase & 1 & 3.1594 & 6.15299 & 5.21098 & 3.13151 \\
\hline Rv2497c & $p d h A$ & Pyruvate dehydrogenase alpha subunit & 1 & 3.73133 & 4.52197 & 5.04976 & 4.00306 \\
\hline Rv2642 & & ArsR family transcriptional regulator & 1 & 3.76985 & 5.16757 & 4.39006 & 3.93426 \\
\hline Rv2644c & & Hypothetical protein & 1 & 3.36059 & 7.58921 & 5.36796 & 3.51825 \\
\hline Rv2660c & & Hypothetical protein & 1 & 13.43717 & 41.25793 & 67.29882 & 19.6699 \\
\hline Rv2661c & & Hypothetical protein & 1 & 9.23174 & 28.30861 & 52.34967 & 11.04351 \\
\hline Rv2662 & & Hypothetical protein & 1 & 20.62942 & 18.83647 & 14.72059 & 12.88898 \\
\hline Rv2663 & & Hypothetical protein & 1 & 7.61461 & 9.43216 & 8.19525 & 7.3034 \\
\hline Rv2664 & & Hypothetical protein & 1 & 6.24636 & 8.49102 & 7.10191 & 5.60291 \\
\hline Rv2666 & & Truncated transposase IS1081 & 1 & 6.91867 & 13.89339 & 7.89331 & 5.86579 \\
\hline Rv2667 & $\operatorname{clpC2}$ & ATP-dependent protease & 1 & 9.44815 & 17.89662 & 9.64508 & 6.46149 \\
\hline Rv2707 & & Conserved transmembrane protein & 1 & 3.35002 & 5.09024 & 14.83903 & 4.53239 \\
\hline Rv2711 & $i d e R$ & Transcriptional regulatory protein & 1 & 3.48877 & 4.30099 & 6.06795 & 3.83858 \\
\hline Rv2713 & $\operatorname{sth} A$ & Soluble pyridine nucleotide transhydrogenase & 1 & 4.43327 & 6.35516 & 6.80833 & 3.83838 \\
\hline Rv2780 & ald & Secreted L-alanine dehydrogenase ALD & 1 & 5.2891 & 4.65988 & 4.52694 & 4.92656 \\
\hline Rv2814c & & Transposase & 1 & 3.3279 & 5.52338 & 4.86873 & 4.60102 \\
\hline Rv2815c & & Transposase & 1 & 3.13667 & 6.24306 & 5.87423 & 4.84337 \\
\hline Rv3185 & & Transposase & 1 & 3.58899 & 6.43621 & 5.67335 & 5.82686 \\
\hline Rv3186 & & Transposase & 1 & 3.2903 & 6.21375 & 6.14822 & 5.77427 \\
\hline Rv3290c & lat & L-lysine aminotransferase & 1 & 4.32023 & 5.06387 & 3.54801 & 3.9704 \\
\hline Rv3474 & & Transposase IS6110 & 1 & 3.04947 & 6.19754 & 6.19869 & 3.22266 \\
\hline Rv3475 & & Transposase IS6110 & 1 & 3.73966 & 5.79892 & 5.63617 & 6.23465 \\
\hline Rv3580c & cys $S$ & Cysteinyl-tRNA synthetase & 1 & 3.87797 & 6.67899 & 3.14124 & 3.40852 \\
\hline Rv3582c & $i s p D$ & 2-C-methyl-D-erythritol 4-phosphate cytidylyltransferase & 1 & 3.50012 & 4.07861 & 3.78626 & 3.51221 \\
\hline
\end{tabular}


During transition to the NC state, some genes used as markers of stress conditions were induced: the heat-shock protein $h s p$, the chaperones Rv0440 and Rv3417c, as well as sigma-factors: sigG - regulating genes which are necessary for survival inside the macrophages and $\operatorname{sig} B$, which can control stationary phase regulons and general resistance to stress. Induction of $\operatorname{ccs} A$, whose product takes part in the cytochrome biosynthesis at the step of heme attachment, and cyp132, coding one of the cytochrome's P450 oxidizing different xenobiotics, could evidently reflect accumulation of toxic components in cultures during transition. Enzymes of the non-mevalonate pathway of isoprenoid biosynthesis ispF and $i s p D$ were also induced in the NC cells. There are data indicating that some of the metabolites of this pathway can affect the immune response of the host [6]. A number of induced genes are involved in the information pathways and those encoding regulatory proteins; in particular, the transcriptional regulator fur $A$, which acts as a global negative control element, employing $\mathrm{Fe}^{2+}$ as a cofactor to bind the operator of the repressed genes. It seems to regulate the transcription of $k a t G$, which is induced in the NC state. katG encodes a multifunctional enzyme, exhibiting both catalase, a broad-spectrum peroxidase and peroxynitritase activities and is believed to play a role in the intracellular survival of mycobacteria within macrophages, protecting them against the aggressive components produced by phagocytic cells. Some genes taking part in the cell wall and cell processes, in particular the transporters ctpG and ctpC encoding ATPases-transporting metal cations and the transporter Rv2688c involved in antibiotic resistance and export of antibiotics across the membrane, are activated.

To identify the genes that were significantly upregulated during transition to the NC state, we used stringent criteria: the expression level during the whole time course in the stationary phase was upregulated at least 3-fold in comparison to the expression in the logarithmic phase. Fifty-one genes met this criterion (Table 2).

Among the genes with a substantially high level of expression, genes encoding insertion sequences and phages 20 genes out of the 51 - are prime candidates, while 13 genes encode hypothetical proteins with unknown function. It is remarkable that the significantly upregulated genes belonged to intermediary metabolism and the respiration category. Moreover, these genes mainly encode proteins involved in degradation processes; namely bgl $S$ - beta-glycosidase (hydrolyzes the terminal, non-reducing beta-D-glucose residue); pip - proline iminopeptidase (specifically catalyses the removal of $\mathrm{N}$-terminal proline residues from small peptides); clpC2 ATP-dependent protease; and ald - L-alanine dehydrogenase (catalyses alanine hydrolyze - an important constituent of the peptidoglycan layer). In addition, the $p d h A$ coding the alpha subunit of pyruvate dehydrogenase and taking part in the energetic metabolism and catalyzing the conversion of pyruvate to acetyl-CoA was highly expressed. Significant upregulation of $\operatorname{sth} A$, a soluble pyridine nucleotide transhydrogenase that catalyses the conversion of NADPH generated by catabolic pathways to $\mathrm{NADH}$, which is oxidized by the respiratory chain for energy generation, is a sign of the prevalence of catabolic reactions in cell metabolism in the NC state.
Analysis of the global gene expression profile has been published for several $M$. tuberculosis persistence models, in particular the Wayne model of the non-replicating state during hypoxia $[5,7,8]$, the gradual depletion of the carbon source under decreased oxygen tension [9], the adaptation of M. tuberculosis within macrophages [10], and in vivo within artificial granulomas in mice [11]. Considering the results of these studies, the gene expression profile in our model of "non-culturability" in the stationary phase has, evidently, some overlaps with the above-mentioned models of persistence (Table 3).

Little in common was found between the genes induced in our model of "non-culturability" and the Wayne dormancy model during hypoxia (Table 3). The Wayne model is characterized by the induction of genes of the dormancy survival regulon (Dos-regulon), a group of 49 genes under the control of devR which codes the regulatory part of the two-component system. Upregulation of the Dos-regulon was found not only for dormant cells under hypoxia in vitro, but also for $M$. tuberculosis cells within macrophages [10], and in the artificial granulomas in mice [11]. In our model of $M$. tuberculosis transition to the NC state in the stationary phase, only two genes from Dos-regulon - Rv0571c and Rv2631 - were found upregulated. Dos-regulon induction was not found in the persisting cells during starvation [12], and only two genes of Dos-regulon were activated during persistence at gradual depletion of the carbon source [9].

A recently published paper [13] demonstrated that the role of Dos-regulon is apparently overestimated not only as a universal regulator of the dormant state of mycobacteria, but also as a general response on hypoxia. Genes of the Dosregulon were shown to be activated only 2 hours after hypoxia.

Table 3. Comparison of genes upregulated during transition to the NC state in the stationary phase (at least 1.5 -fold) to the genes activated in other models of persistence

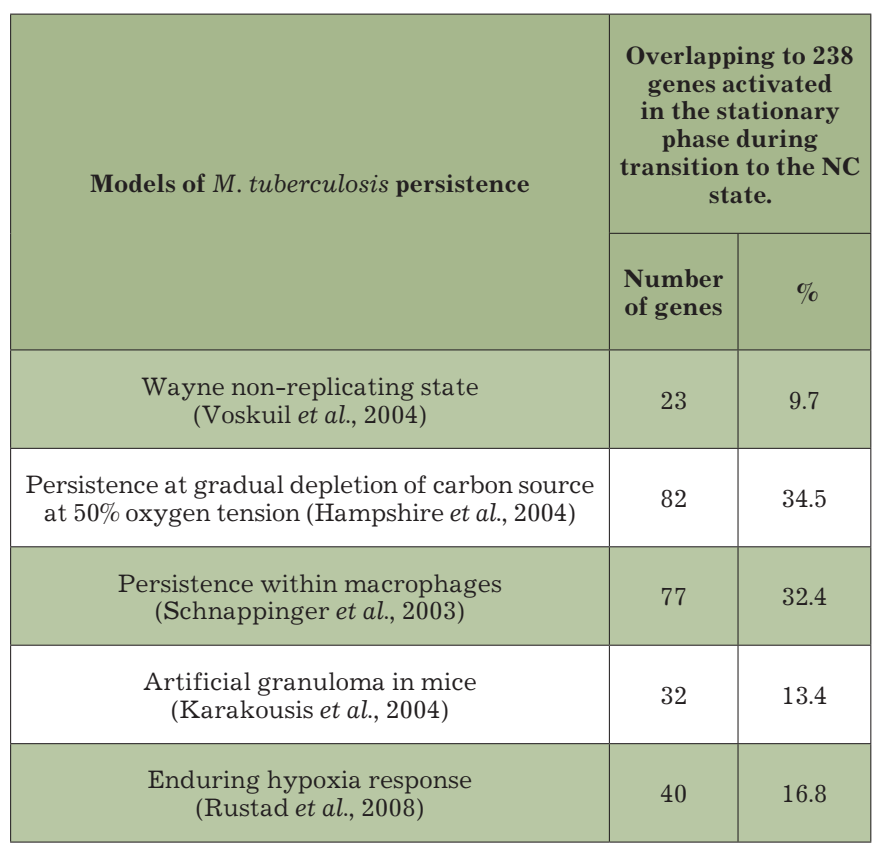


Table 4. Shared genes of M. tuberculosis persistence state. Genes of EHR regulon are in bold

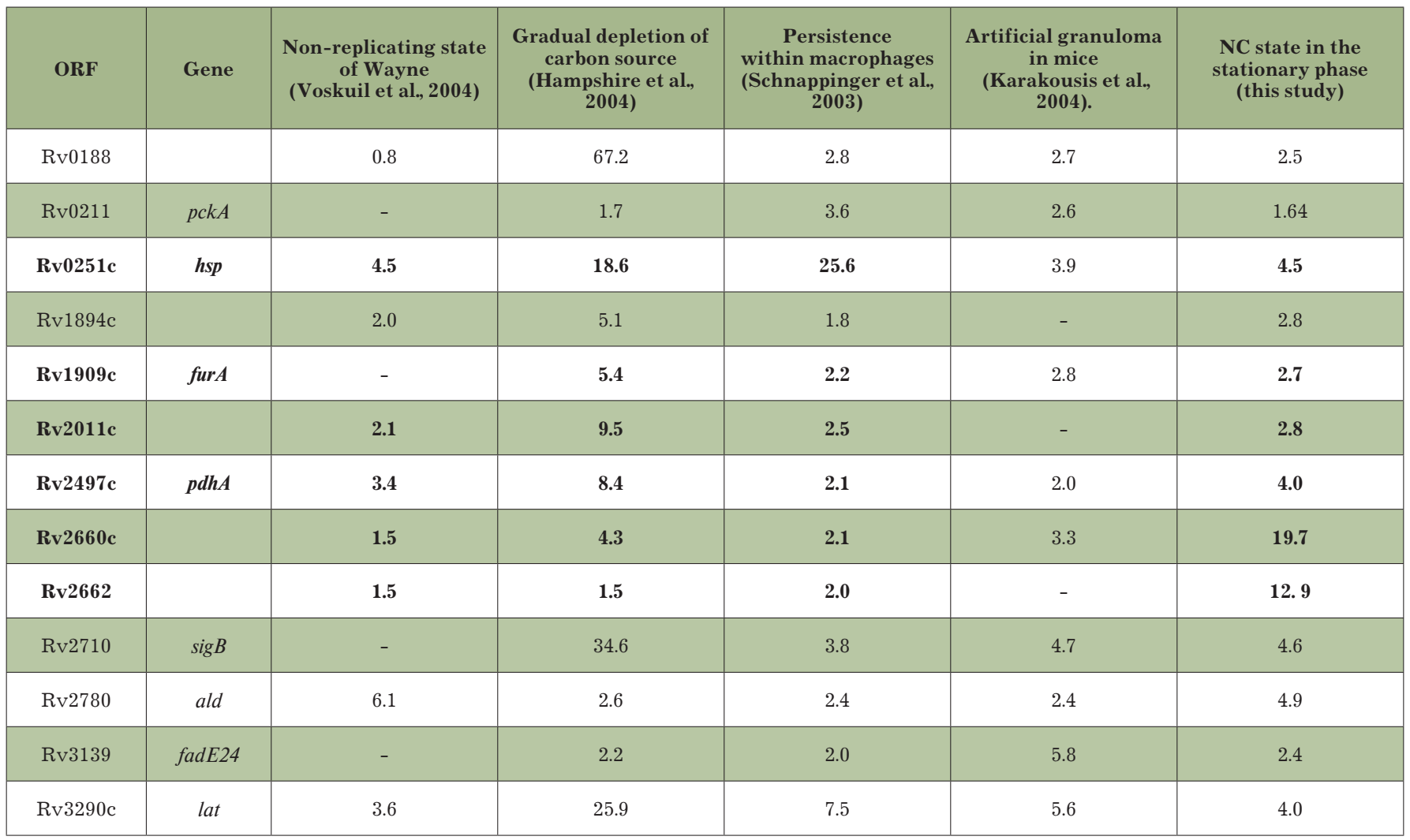

Thereafter expression of at least half of these returned to the baseline [13]. The authors observed a significant induction of another 230 genes after further cultivation during hypoxia, and hereafter their expression level was stable. Thus, the authors refer to this group of genes as enduring hypoxia response (EHR) genes. Considering the gene expression profile for our model of transition to the NC state, we found significant overlap with this group of genes (Table 3), which was rather unexpected because the conditions for $\mathrm{NC}$ cell formation developed in our laboratory did not imply any oxygen limitation. Some overlap with EHR [13] was found for the persistence model of gradual depletion of the carbon source [9] and the transcriptional response to multiple stresses [14]. Therefore, it is possible to conclude that EHR genes may not only play a role as hypoxia markers, but may also be a general regulon of the dormant state of M. tuberculosis, independent of its induction.
Thus, the data presented here indicate that cell transition to dormant state is an active process and that numerous genes are involved in it. The future task is to investigate this process in detail in order to understand the molecular mechanisms in the cells during the transition to the dormant state.

Based on the results of the transcriptome analysis of the $\mathrm{NC}$ cells obtained in our model and those obtained in several models of persistence, it is possible to pinpoint some shared genes that are upregulated in these models (Table 4). The genes presented in Table 4 and their products are believed to be important for further study, because some of them could represent new targets for anti-tuberculosis drug candidates directed mainly against latent tuberculosis.

This work was supported by the Program of the Presidium of the RAS "Molecular and Cellular Biology"

\section{REFERENCES}

1. Gangadharam P. R. J. Tuber. Lung Dis., 1995, 76, 477-479.

2. Wayne L. G. and Lin K.-Y. Infect. Immun., 1982, 37, 1042-1049.

3. Wayne L. G. and Hayes L. G. Infect. Immun., 1996, 64, 2062-2069.

4. Mukamolova G. V., Salina E. G., Kaprelyants A. C. in National Institute of Allergy and Infectious Diseases, NIH (Georgiev, V., ed), Humana Press, USA, 2008, 1, 83-90.

5. Voskuil M. I., Visconti K. C., Schoolnik G. K. Tuberculosis (Edinb.), 2004, 84, 218-227.

6. Shao L., Zhang W., Zhang S., Chen CY., Jiang W., Xu Y., Meng C., Weng X., Chen Z.W. AIDS, 2008, 22(17), 2241-508.

7. Muttucumaru D. G., Roberts G., Hinds J., Stabler R. A., Parish, T. Tuberculosis (Edinb.), 2004, 84, 239-246.

8. Bacon J., James B. W., Wernisch L., Williams A., Morley K. A., Hatch G.J., Mangan J.A.,
Hinds J., Stoker N.G., Butcher P.D., and Marsh P.D. Tuberculosis (Edinb.), 2004, 84, 205-217 9. Hampshire T., Soneji S., Bacon J., James B. W., Hinds J., Laing K., Stabler R. A., Marsh P. D., and Butcher P. D. Tuberculosis (Edinb),2004, 84, 228-238.

10. Schnappinger D., Ehrt S., Voskuil M. I., Liu Y., Mangan J. A., Monahan I. M., Dolganov G., Efron B., Butcher P. D., Nathan C., Schoolnik G. K. J. Exp Med., 2003, 198(5), 693-704. 11. Karakousis P. C., Yoshimatsu T., Lamichhane G., Woolwine S. C., Nuermberger E.L., Grosset J., Bishai, W. R. J. Exp. Med., 2004, 200, 647-657.

12. Betts J. C., Lukey P. T., Robb L. C., McAdam R. A., and Duncan K. Mol. Microbiol., 2002, 43, 717-731.

13. Rustad T. R., Harrell M. I., Liao R, Sherman D. R.. PLoS ONE, 2008, 3(1), 1502.

14. Boshoff H. I., Myers T. G., Copp B. R., McNeil M. R., Wilson M. A., and Barry C. E., 3rd J. Biol. Chem., 2004, 279, 40174-40184. 